\title{
Review Article \\ Optical Second Harmonic Generation Microscopy as a Tool of Material Diagnosis
}

\author{
Hiroko Yokota, ${ }^{1,2}$ Junichi Kaneshiro, ${ }^{1}$ and Yoshiaki Uesu ${ }^{1}$ \\ ${ }^{1}$ Department of Physics, Waseda University, Okubo, Shinjuku-Ku, Tokyo 169-8555, Japan \\ ${ }^{2}$ Department of Physics, Chiba University, Yayoi-Cho, Inage, Chiba 263-8522, Japan \\ Correspondence should be addressed to Yoshiaki Uesu, uesu93@waseda.jp
}

Received 31 December 2011; Accepted 12 March 2012

Academic Editor: Tatiana V. Murzina

Copyright ( 2012 Hiroko Yokota et al. This is an open access article distributed under the Creative Commons Attribution License, which permits unrestricted use, distribution, and reproduction in any medium, provided the original work is properly cited.

\begin{abstract}
The second harmonic generation microscope (SHGM) constructs images of intensity distributions of SH waves produced by the interaction of fundamental waves with a polar material. We have developed this nonlinear optical microscope in order to make possible nondestructive, three-dimensional (3D) observations of various kinds of inorganic and organic materials. The SHGM can disclose also inverted domain structures of antiparallel spontaneous polarizations using the interference with the reference SH waves. The observation principle and several applications to structural characterizations of $\mathrm{LiNbO}_{3}$ and $\mathrm{LiTaO}_{3}$ quasi-phase matching devices, domain structure analyses of a relaxor/ferroelectric solid solution $\mathrm{Pb}\left(\mathrm{Zn}_{1 / 3} \mathrm{Nb}_{2 / 3}\right) \mathrm{O}_{3}-9 \% \mathrm{PbTiO}_{3}$ at the morphotropic phase boundary, development of order parameter in a quantum paraelectric relaxor $\mathrm{Li}$-doped $\mathrm{KTaO}_{3}$, and antiphase polar domain structures of muscle fibers and myofibrils are surveyed by stressing the high effectiveness of the SHGM as a tool of material diagnosis.
\end{abstract}

\section{Introduction}

Optical second harmonic generation (SHG) is a phenomenon where coherent light with twice the fundamental frequency is generated through the nonlinear interaction between a laser and a material. Since SHG is sensitive to the break of spatial inversion (SI) symmetry or time inversion (TI) symmetry, it provides a powerful experimental technique for investigating ferroelectricity related to the SI symmetry break [1-3], magnetic ordering related to the TI symmetry break $[4,5]$, and multiferroics related to both phenomena [6-9].

Certain kinds of materials are classified as ferroics where spontaneous electric polarization $P_{s}$ (ferroelectrics), spontaneous magnetic order $M_{s}$ (ferromagnetics), or spontaneous strain $x_{s}$ (ferroelastics) can be reversed by their conjugate external fields $E, H$, or $X$, respectively. Ferroics have a phase transition temperature $T_{c}$. Above $T_{c}$ these properties disappear, while below $T_{c}$, the spontaneous order parameters develop in macroscopic or mesoscopic regions having different orientations but same magnitude of spontaneous values. These regions are termed domains or variants. Spontaneous order parameter displays a hysteresis loop upon applying these conjugate external fields and these phenomena are the process of changing in domain states from multiple to single domain and vice versa. Recently much attention has been paid to cross-correlations of $P_{s}-H$ and $M_{s}-E$ in multiferroics and a lot of studies have been performed in this promising field.

In the case of ferroelectrics, the development of $P_{s}$ can be detected by SHG in a wide dynamic range to determine the critical index at the vicinity of the phase transition point $[10,11]$. SHG has also been used to observe domain structures by exploiting the spatial distributions of the SHG tensor component [12-15]. Based upon this advantage of SHG for material characterizations, we have developed the SHG microscope (SHGM) for material characterization, in particular, nondestructive and three-dimensional (3D) observations of domain structures of inorganic, organic, and biological materials. This paper reviews the principle of our SHGM and its application to observations of domain structures in certain kinds of ferroelectrics and biological tissues of muscles as well. Section 2 describes the principle of SHG (Section 2.1), the principle of SHG interference 
microscopy for observing antiparallel domain structure (Section 2.2), an intrinsic issue ( $\Delta k$ issue) for realizing $3 \mathrm{D}$ domain structures using the strongly focused scanning-type SHGM (Section 2.3), and the polar diagram representation of SHG images and related problems (Section 2.4). Section 3 explains the optical system of our microscope. Section 4 shows some examples of SHG images and their analyses picking up 4 cases: 3D observations of periodically poled domain (PPD) structures in quasi-phase matching (QPM) devices for wavelength converters of lasers (Section 4.1), complex domain structures of a relaxor/ferroelectric mixed solution $\mathrm{Pb}\left(\mathrm{Zn}_{1 / 3} \mathrm{Nb}_{2 / 3}\right) \mathrm{O}_{3}-9 \% \mathrm{PbTiO}_{3}$ (PZN/9PT) at the morphotropic phase boundary (MPB) (Section 4.2), a quantum paraelectric relaxor $\mathrm{K}_{x} \mathrm{Li}_{(1-x)} \mathrm{TaO}_{3}$ (KLT) (Section 4.3), and muscle fibers and myofibrils (Section 4.4). Section 4 sums up the results and discusses future trends of SHG microscopy.

\section{Theoretical Treatment of SHG}

2.1. Principle of SHG. As a result of the nonlinear coupling of fundamental wave fields and materials, the following nonlinear wave propagation equation (1) is derived from the Maxwell equation. SHG is a case of the second-order nonlinear optical process where $P_{\mathrm{NL}}$ is proportional to the square of the fundamental wave fields. As a result, coherent waves with the frequency twice, therefore, the wavelength half of the fundamental waves are generated:

$$
\nabla^{2} E-\varepsilon \mu \frac{\partial^{2} E}{\partial t^{2}}=\mu \frac{\partial^{2} P_{\mathrm{NL}}}{\partial t^{2}}+\mu_{0} \nabla \times \frac{\partial M_{\mathrm{NL}}}{\partial t}-\mu \frac{\partial^{2}(\nabla Q)}{\partial t^{2}} .
$$

Here the right hand terms express the light source of harmonic waves: $P_{\mathrm{NL}}$ is the nonlinear polarization induced by the fundamental laser beam, $M_{\mathrm{NL}}$ is the nonlinear magnetization, $Q$ is the electric quadrupole moment, and $\varepsilon$ and $\mu$ are dielectric and magnetic susceptibilities in a sample, respectively. In general cases, the first term is dominant in the SHG, and we consider only this term in the following. The left hand terms express the propagation of waves generated by the right hand terms.

$P_{\mathrm{NL}}\left(=P^{(2 \omega)}\right)$ and the electric field $E^{(\omega)}$ of the fundamental wave are related through the SHG tensor component $d_{i j k}$ $(d$-constant $)$ as is expressed in the following equation:

$$
P_{k}^{(2 \omega)}=\varepsilon_{0}\left(d_{k l m}^{(i)}+d_{k l m}^{(c)}\right) E_{l}^{(\omega)} E_{m}^{(\omega)} .
$$

Here $d^{(i)}$ and $d^{(c)}$ mean the third-rank tensors and $d^{(i)}$ is invariant and $d^{(c)}$ is variant with respect to the time inversion operation. These $d$-constants are related to $P_{s}$ and magnetic order parameter $F_{m}$ as

$$
d^{(i)}=d(0)+\alpha P_{s}, \quad d^{(c)}=\beta F_{m}+\gamma P_{s} F_{m} .
$$

The relation of these constants to the existence of spatially inversion and magnetic inversion symmetries is summarized in Table 1. Since SHG intensity is proportional to the square of $d$-constant, the behavior of structural and magnetic order parameters and the correlation between them can be obtained from SHG measurements. Hereafter we describe

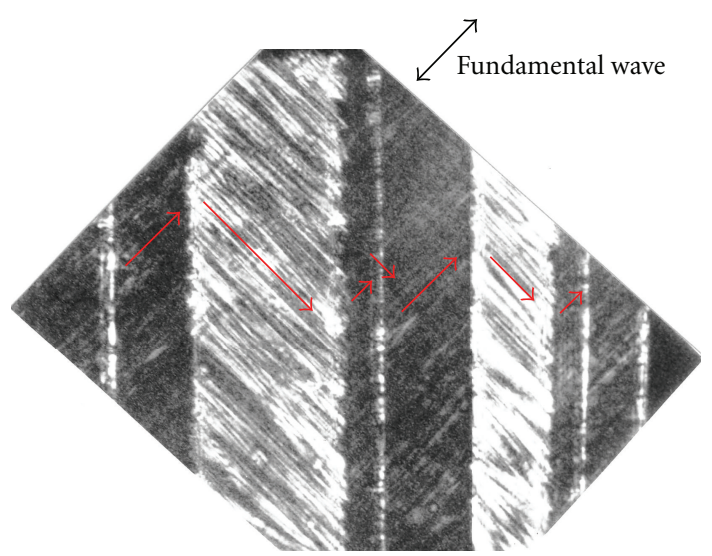

Figure 1: SHG image of $\mathrm{BaTiO}_{3}$ taken by the noninterference technique. The polarization direcion of the fundamental wave is fixed along the black arrow. $90^{\circ}$ domain structure is observed as a bright and dark regions. Red allows indicate the direction of $P_{s}$.

phenomena related to $d^{(i)}$ in nonresonant wavelength region, that is, the case of noncentrosymmetric and nonmagnetically ordered transparent materials.

For getting the SH wave field from (1), we consider the collinear case where the fundamental wave with $\mathrm{TEM}_{00}$ Gaussian mode with a beam radius $\omega_{0}$ at the focal position and the Rayleigh length $z_{0}$ and $\mathrm{SH}$ waves propagate along the $z$ axis. The complex field amplitude of the $\mathrm{SH}$ wave generated in a sample is obtained as [16]

$$
\Psi \propto \int_{0}^{L} \frac{d \exp (i \Delta k z-\alpha z)}{z-l_{f}+i z_{0}} \mathrm{~d} z
$$

Here $\Delta k$ is a measure of the wave number offset between the fundamental $\left(k_{1}\right)$ and $\mathrm{SH}$ waves $\left(k_{2}\right)$ and is expressed by

$$
\Delta k=k_{2}-2 k_{1}
$$

$L$ is the sample thickness and $l_{f}$ is the distance of the focal position from the incident surface of the sample. $\alpha$ is an absorption parameter expressed as $\alpha^{\omega}-(1 / 2) \alpha^{2 \omega}$, and $\alpha^{\omega}$ and $\alpha^{2 \omega}$ are the intensity absorption coefficients of the fundamental and $\mathrm{SH}$ waves, respectively. In a sample with multidomain structures, $d, \Delta k, \alpha$, and $z_{0}$ are dependent on the spatial coordinates $(x, y, z)$.

\subsection{Principle of SHG Interference Microscopy for Observing} Antiparallel Domain Structures. A simple and general method of observing domain structures by SHG is to exploit the anisotropy of $d$-constants. For example, $90^{\circ}$ domain structures with $P_{s}$ directed along the [100], [010] and [001] axes in ferroelectric tetragonal $\mathrm{BaTiO}_{3}$ can be observed using the difference in magnitude of $d_{311}$ and $d_{333}$ as shown in Figure 1 [12]. However, $180^{\circ}$ domain with antiparallel $P_{s}$ cannot be distinguished, because $\mathrm{SH}$ waves have same amplitude for both domains. Other optical method cannot also detect this domain structure. The nondestructive observation of $180^{\circ}$ domain structure has been required in particular to 
TABLE 1: The relation of $d$ constants to the existences of spatial and magnetic inversion symmetries.

Time reversal

$\times$

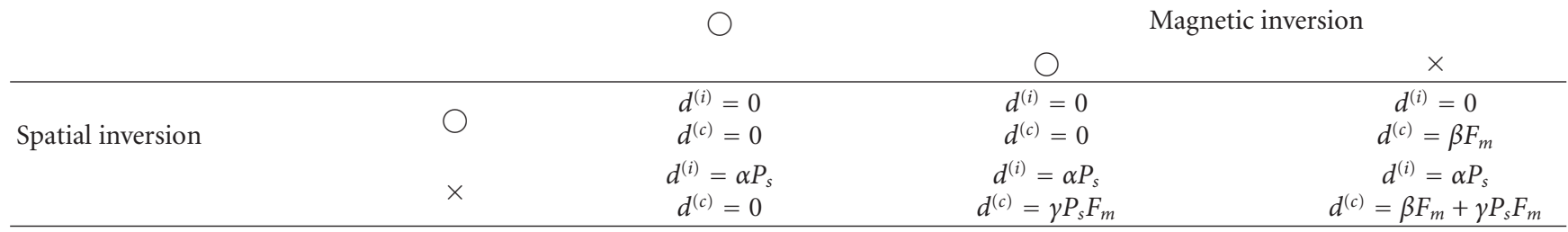

$\bigcirc$ : the case of existence, $\times$ : the case of absence.

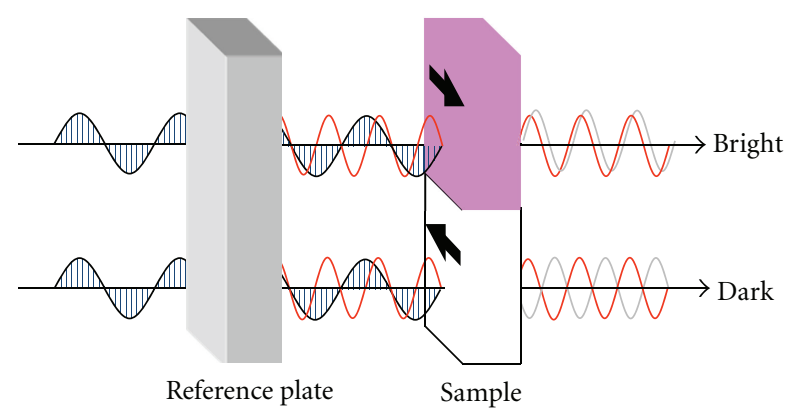

$\longrightarrow$ Fundamental waves

$\longrightarrow$ Reference waves

$\longrightarrow \mathrm{SH}$ waves from the sample

FIGURE 2: Observation principle of antiparallel domain structures. Homogeneous SH wave generated from a reference plate is mixed constructively with $\mathrm{SH}$ waves from +domain and destructively in -domain of the sample.

characterize precisely QPM devices. This motivated us to develop the SHG interference microscope (SHGIM) that makes the observation of $180^{\circ}$ domain structure possible $[12,13,17,18]$.

The SHG interference microscope (SHGIM) exploits the interference of $\mathrm{SH}$ waves generated in a sample and a reference plate to observe $180^{\circ}$ domain structures in ferroelectrics. The principle is schematically illustrated in Figure 2, where a sample contains two domains with opposite polarities. In this case, the signs of $d$-constants are opposite and the phase difference of $\mathrm{SH}$ waves generated in these two domains is $\pi$. Therefore, with uniform reference waves, the interference produces an intensity contrast. This principle of SHGIM can be described analytically as below in a strongly focusing optical system, such as the present optical system.

Equation (4) is modified when up $\left(+d_{i j k}\right)$ and down $\left(-d_{i j k}\right)$ domains exist in a sample as

$$
\Psi^{( \pm)}= \pm d_{i j k} \int_{0}^{L} \frac{\exp i \Delta k z}{z-l_{f}+i z_{0}} \mathrm{~d} z
$$

The interference intensity of $\Psi^{( \pm)}$and the reference SH wave $\Psi_{\text {ref }}$ are described by

$$
\begin{aligned}
\mid \Psi^{( \pm)}+ & \left.\Psi_{\text {ref }} \exp (-i \varphi)\right|^{2} \\
& =\left|\Psi^{( \pm)}\right|^{2}+\left|\Psi_{\text {ref }}\right|^{2}+2 \operatorname{Re}\left\{\Psi^{( \pm)} \Psi_{\text {ref }}^{*} \exp i \varphi\right\} .
\end{aligned}
$$

Here, $\varphi$ is the phase difference between the fundamental and $\mathrm{SH}$ waves. Because $\Psi^{( \pm)}$is proportional to $d_{i j k}$, the interference intensities, the third term in the right-hand side, of antiparallel domains are different, and the $180^{\circ}$ domain structure can be observed as the intensity contrast. The phase $\varphi$ is varied to obtain the maximum intensity contrast by a phase modulator.

2.3. Principle of Observations of Internal Domain Structures: $\Delta k$ Issue. Our SHGIM enables us to observe polar domain structures in cross-sections of a sample. For this 3D observation, we adopt a confocal optical system composed of a pair of objective lenses with high numerical aperture (NA) and a pinhole (see the optical system of SHGIM in Section 3). The optical diffraction limit $w_{0}$ at the focal position and the Rayleigh length $z_{0}$ are measures of the spatial resolutions along the lateral and axial directions, respectively, and both characteristic lengths are related to NA as

$$
w_{0}=0.61 \frac{\lambda}{\mathrm{NA}}, \quad z_{0}=\frac{\pi n_{1} w_{0}^{2}}{\lambda} .
$$

Here, $\lambda$ is the wavelength of an incident wave, and $n_{1}$ is the refractive index of a sample at $\lambda$. Since $w_{0}$ and $z_{0}$ are proportional to $(\mathrm{NA})^{-1}$ and $(\mathrm{NA})^{-2}$, respectively, a higher NA yields higher spatial resolutions. To describe the degree of focus, a nondimensional parameter $\xi$ is introduced:

$$
\xi=\frac{L}{2 z_{0}} .
$$

It should be noted that the strongly focused optical system with high NA yields phenomena substantially different from a weakly focused one, which affects the visibility of domain structures on the inside of a sample [19].

In the weakly focused case $(\xi \ll 1)$, the plane wave approximation is valid and the $\mathrm{SH}$ intensity is expressed as

$$
I^{2 \omega} \propto L^{2} \frac{\sin ^{2}((1 / 2) \Delta k L)}{((1 / 2) \Delta k L)^{2}} .
$$




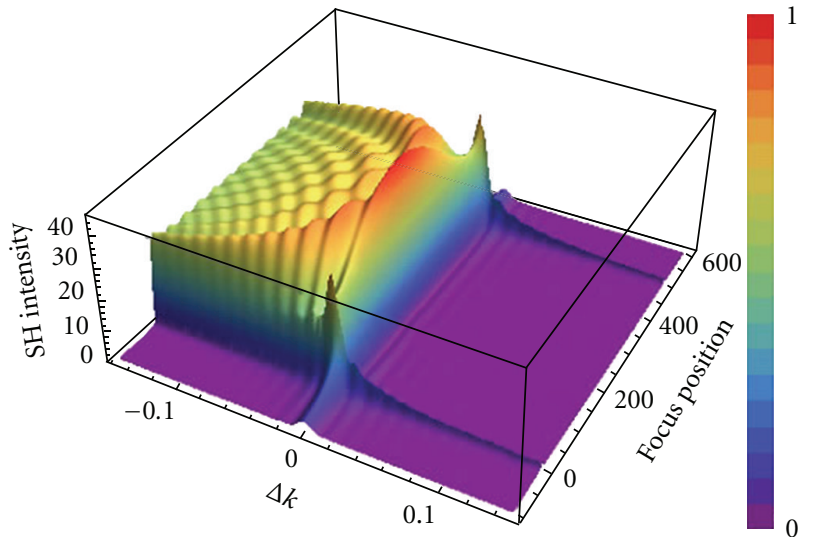

FIGURE 3: Simulated SHG intensity as functions of the focus position and $\Delta k$. Here, the sample thickness is set as $L=500 \mathrm{~nm}$. SHG intensity from inside of a sample is almost null in the case of $\Delta k>0$. For $\Delta k<0, \mathrm{SH}$ waves are generated also from inside of the sample [19].

Equation (10) indicates that $I^{2 \omega}$ does not depend on the sign of $\Delta k$. However, when $\xi$ becomes larger, $I^{2 \omega}$ cannot be approximated by (10) and depends strongly on the sign of $\Delta k$ and the focus position as shown in Figure 3 [19]. Let us call this phenomenon " $\Delta k$ issue," stressing the important observation criterion of the internal domain structures using SHGM. This simulation result is calculated using (4) with similar magnitudes as in the present experiments. The SHG intensity $I^{2 \omega}$ decreases drastically inside the sample when $\Delta k$ changes from negative to positive. On the other hand, when the focus position is located in the vicinity of end surfaces, $I^{2 \omega}$ remains large and decreases slowly with $\Delta k$. This phenomenon induces the degradation of the visibility of inside structures in the case of positive $\Delta k$ [16] and the use of $d$-constants with negative $\Delta k$ is strongly requested.

The physical origin of the $\Delta k$ issue is explained as follows. Generally, the phase of Gaussian wave changes from $-\pi / 2$ to $+\pi / 2$ upon propagation. This phase is known as the Gouy phase and is largely changed at the focus point: the more strongly focused is the wave, the larger is the change in the phase at the focus position. This phase change makes the effective phase $\Delta K$ of SH waves toward the positive direction and makes away the phase-matching condition. On the other hand, negative $\Delta k$ of the sample pulls the effective phase toward $\Delta K=0$ which can satisfy the phase match condition to obtain large SHG intensities.

2.4. Polar Diagram Representation of SHG Images. For quantitative analyses of SHG images, 2D maps of polarization dependences of SHG intensity in the polar diagram (PolD) are measured for each area in mesh under the condition that the polarization directions of the incident and $\mathrm{SH}$ waves are kept parallel to each other [20]. The result is fitted with calculated values that are obtained from the domain configurations. The following equations show the case of PZN/9PT under the assumption of the monoclinic space group Pm. In this calculation, we adopt the laboratory coordinates $(x, y, z)$ parallel to the cubic axes with the fundamental wave incident along the $y$ axis and the $\mathrm{SH}$ intensities produced in the $z x$ plane. The SHG intensities are expressed as a function of the polarization angle $\theta$ :

Type 1:

$$
\begin{aligned}
I_{1}^{2 \omega}(\theta) \propto[ & {\left[\left(\chi_{x x x} \cos ^{2} \theta+\chi_{x z z} \sin ^{2} \theta+2 \chi_{x z x} \sin \theta \cos \theta\right) \cos \theta\right.} \\
& \left.+\left(\chi_{z x x} \cos ^{2} \theta+\chi_{z z z} \sin ^{2} \theta+2 \chi_{z z x} \sin \theta \cos \theta\right) \sin \theta\right]^{2},
\end{aligned}
$$

Type 2:

$$
I_{2}^{2 \omega}(\theta) \propto\left[\left(\chi_{x x x} \cos ^{2} \theta+\chi_{x z z} \sin ^{2} \theta\right) \cos \theta+2 \chi_{z z x} \cos \theta \sin ^{2} \theta\right]^{2},
$$

Type 3:

$$
I_{3}^{2 \omega}(\theta) \propto\left[2 \chi_{x z x} \cos ^{2} \theta \sin \theta+\left(\chi_{z x x} \cos ^{2} \theta+\chi_{z z z} \sin ^{2} \theta\right) \sin \theta\right]^{2} .
$$

In the monoclinic system, the twenty-four spontaneous polarization states exist and the observed PolDs are classified into three groups, expressed by (11)-(13). Types 1-3 correspond to the classification of Table 2 , and the form of PolD is determined by the direction of $\mathbf{P}_{s}$ projected onto the $z x$ plane. The results of PZN/9PT are discussed in Section 4.2.

\section{Optical System of SHGIM}

The optical system of the SHGIM is illustrated in Figure 4. The light source (1) is an $\mathrm{Nd}: \mathrm{YVO}_{4}$ laser with a wavelength of $1064 \mathrm{~nm}$, a pulse width of $10 \mathrm{~ns}$, a repetition rate of $40 \mathrm{kHz}$, and a maximum power of $6 \mathrm{~W}$. The fundamental beam is expanded by a couple of lenses $(2 a$ and $2 b)$ and divided into two beams by a separator (3). One is used for changing the phase of the fundamental wave by a phase modulator (7), and the other is for producing the reference SH waves by a QPM wave converter (9). Intensities of the fundamental and reference SH waves can be changed independently by attenuators composed of a half wave plate and a thin-film polarizer $\left(4\right.$ and $\left.4^{\prime}\right)$. The polarization direction of the incident waves is changed by a half-wave plate (5). Fundamental and reference $\mathrm{SH}$ waves are focused to a sample by an objective (12a) with a numerical aperture (NA) of 0.7. Average fluence of the laser beam at the sample position is less than $30 \mathrm{~J} / \mathrm{cm}^{2}$ so as to produce no optical damage in samples. Total $\mathrm{SH}$ waves generated from the sample and the reference plate pass through a rear objective (12b), and the polarization direction of the $\mathrm{SH}$ waves is selected by an analyzer (15). The light with wavelength $532 \mathrm{~nm}$ is selected by a spectrometer (18), and the intensity is detected point by point by a photomultiplier tube (19) that is synchronized with incident laser pulses by a lock-in amplifier (20). The sample stage (13) is driven by the combination of stepping motors and piezoactuators (14) along the three mutually perpendicular directions. Sectional SHG images are 


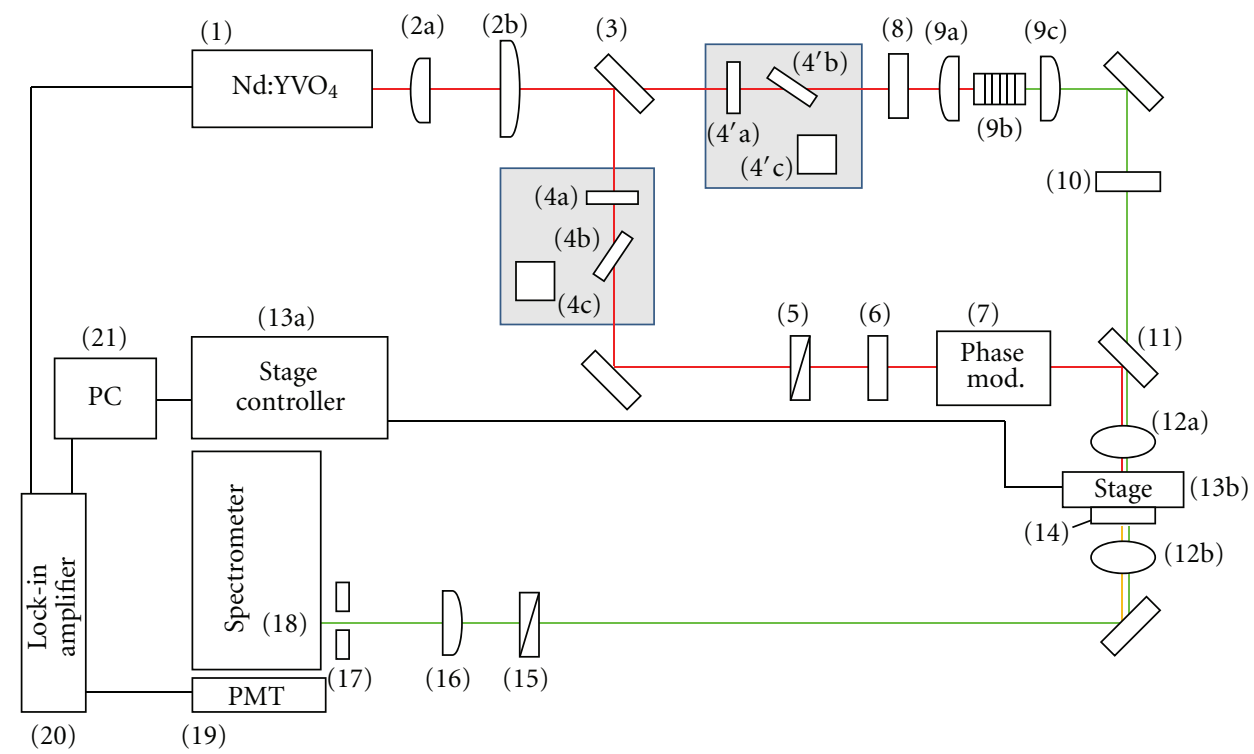

Figure 4: The optical system of SHGIM; (1) Nd: YVO laser, (2a, b) lens, (3) a separator, $\left(4,4^{\prime}\right)$ an attenuator, (5) a polarizer, $(6,8,10)$ a half-wave plate, (7) a phase modulator, (9) a QPM wave-converter, (11) a harmonic separator, (12) objectives with NA of 0.7, (13) a stepping motor sample stage, (14) a piezo-actuator stage, (15) an analyzer, (16) a lens, (17) an aperture variable pinhole, (18) a spectrometer, (19) a photomultiplier tube, (20) a lock-in amplifier, (21) a computer.

TAble 2: Possible directions of $P_{s}$ in monoclinic $P m$ system. Here $p_{1}>p_{3}>0$ [20].

\begin{tabular}{lll}
\hline \multirow{2}{*}{ Type 1 } & $\pm \mathbf{P}_{1}=\left( \pm p_{1}, 0, \pm p_{3}\right)$ & $\pm \mathbf{P}_{2}=\left( \pm p_{3}, 0, \pm p_{1}\right)$ \\
& $\pm \mathbf{P}_{3}=\left( \pm p_{3}, 0, \mp p_{1}\right)$ & $\pm \mathbf{P}_{4}=\left(\mp p_{1}, 0, \pm p_{3}\right)$ \\
Type 2 & $\pm \mathbf{P}_{5}=\left( \pm p_{1}, \mp p_{3}, 0\right)$ & $\pm \mathbf{P}_{6}=\left( \pm p_{3}, \mp p_{1}, 0\right)$ \\
& $\pm \mathbf{P}_{7}=\left( \pm p_{3}, \pm p_{1}, 0\right)$ & $\pm \mathbf{P}_{8}=\left( \pm p_{1}, \pm p_{3}, 0\right)$ \\
Type 3 & $\pm \mathbf{P}_{9}=\left(0, \pm p_{3}, \mp p_{1}\right)$ & $\pm \mathbf{P}_{10}=\left(0, \mp p_{3}, \mp p_{1}\right)$ \\
& $\pm \mathbf{P}_{11}=\left(0, \pm p_{1}, \pm p_{3}\right)$ & $\pm \mathbf{P}_{12}=\left(0, \mp p_{1}, \pm p_{3}\right)$ \\
\hline
\end{tabular}

displayed by a computer (21). With this microscope we can select the noninterference geometry simply by shutting off the second path to the reference plate.

We also constructed an SHGIM equipped with an imageintensified CCD. In this system, the 2D SHG image of a sample integrated along the incident direction is observed. This has not high spatial resolution such as the scanning microscope but has a merit of higher temporal resolution of several seconds. We applied this ICCD SHGIM for observing development of the order parameter of KLT (see Section 4.3).

\section{SHG Images and Domain Structure Analyses}

4.1. Periodically Poled Domain Structures in Quasi-Phase Matching Devices. As a first example of SHGM observations, we show here nondestructive and $3 \mathrm{D}$ characterizations of PPD structures in $\mathrm{LiNbO}_{3}$ and $\mathrm{LiTaO}_{3}$ QPM devices for wavelength converters of laser. The wavelength conversion technique is highly demanded in the optoelectronics for obtaining short-wavelength laser sources or optical parametric oscillators. To obtain high conversion efficiency, it requires the phase coincidence of optical waves generated in a nonlinear medium. This is termed phase matching techniques, among which the QPM is most promising because of flexibility of the incident wavelength and possible use of the maximum SHG tensor component. Since the efficiency depends strongly on the exact periodicity of the PPD structure in a designed volume area, nondestructive and $3 \mathrm{D}$ observations have been required in place of a chemical etching technique which damages devises and provides only domain structure at sample surfaces. However, only two methods are reported: one is the two-wave mixing technique based on the photorefractive effect [21] and another uses four SHG interference images with different phases [22]. Both methods have drawbacks: the former method depends on the specific optical property of a sample which is undesirable as a stable wavelength converter, and the latter needs complicate process of analyses.

We apply the strongly focused scanning SHGIM to the observation of cross-section images of the PPD structure in an $\mathrm{MgO}$ : stoichiometric $\mathrm{LiTaO}_{3}$ (MgSLT) QPM [17]. In this study, however, we observe clear images of the PPD structure with the period of $8 \mu \mathrm{m}$ only near surfaces. This is because we use the $d_{22}$ component whose $\Delta k$ is positive. On the other hand, when we observed the PPD structure of a QPM devise fabricated into $\mathrm{MgO}$-doped $\mathrm{LiNbO}_{3}$ using 


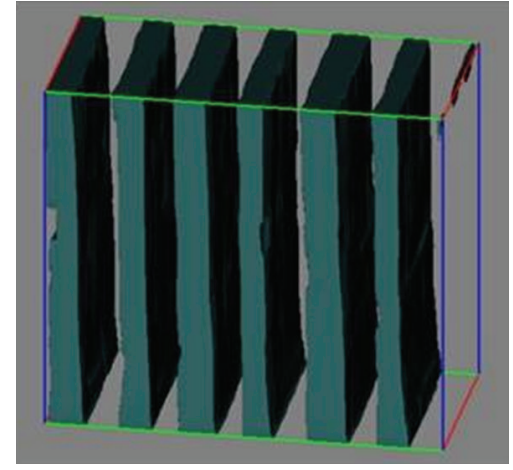

$y=0 \mu \mathrm{m}$

(a)

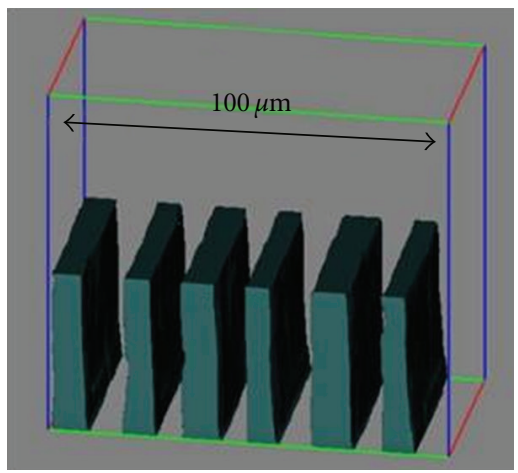

237

(d)

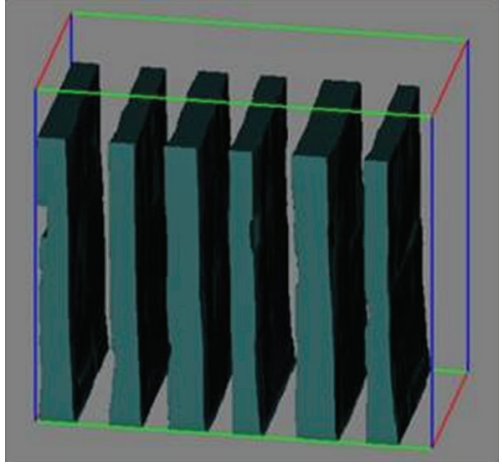

65

(b)

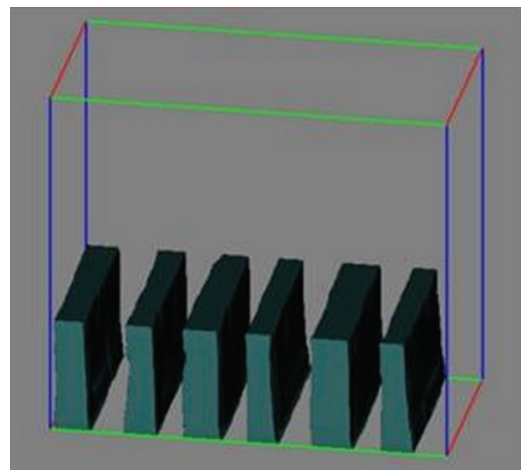

299

(e)

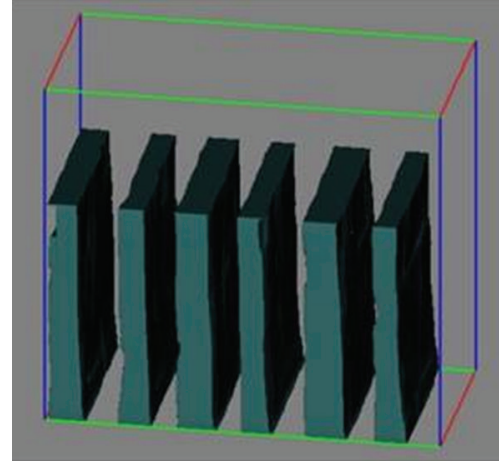

154

(c)

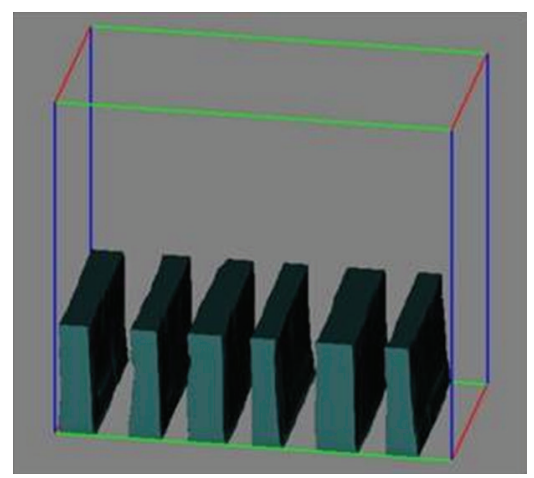

388

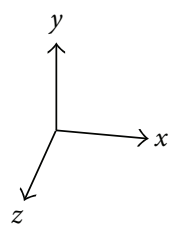

(f)

FIGURE 5: 3D SHG interference images of a QPM devise of $\mathrm{MgO}$-doped $\mathrm{LiNbO}_{3}$. Dark regions indicate inverted domains. The number on the top of each figure means the section position measured from the top surface of the sample. The $P_{s}$ direction is along the $c$ axis.

the $d_{31}$ component with negative $\Delta k$, the PPD structure of a designed $19.5 \mu \mathrm{m}$ period is clearly observed inside the sample. The result is shown in Figure 5.

4.2. Domain Structure Analyses of PZN/9PT. High piezoelectric constants and mechanical coupling coefficients are observed in solid solutions of relaxors and ferroelectrics at specific composition ratios. This compositional phase boundary is termed the MPB [23]. The symmetry of the MPB has been found to be lower than those of the two end members (trigonal in relaxor-rich side and tetragonal in ferroelectric rich side) $[24,25]$. This symmetry lowering to the monoclinic phase makes the rotation of $P_{s}$ more facile, which induces greater piezoelectricity [26]. The symmetry of its MPB has been investigated using several kind of experimental method without the definite conclusion. This is the motive of our study of domain structure analyses of PZN/9PT at the MPB using SHG microscopy. We observed the domains with the spatial resolution of the optical diffraction limit of about several hundred $\mathrm{nm}$, and the symmetry was determined by analyzing the $2 \mathrm{D}$ polar diagram maps [20]. In this study, we cannot observe $180^{\circ}$ domain structures because we do not use the SHG interference technique.

Figure 6(a) shows an SHG image of the PZN-9PT (010)c plate. Bright and dark parts represent the area generating strong and weak $\mathrm{SH}$ intensities, respectively. A photograph of the same area taken by the polarization microscope in the crossed Nicols is also shown in Figure 6(b) for comparison. Similar domain structures are observed in both figures. However, the SHG image provides more clearly the domain structure as bright and dark stripe patterns as shown in this figure, and furthermore, more quantitative information can be obtained from SHGM as described below.

Figure 7(a) shows SHG images of the region A taken with finer scanning step and Figure 7(b) shows the PolD maps corresponding to Figure 7(a). It is obtained with parallel $\mathrm{P}$ and A configuration. Two patterns exist indicating blue and 


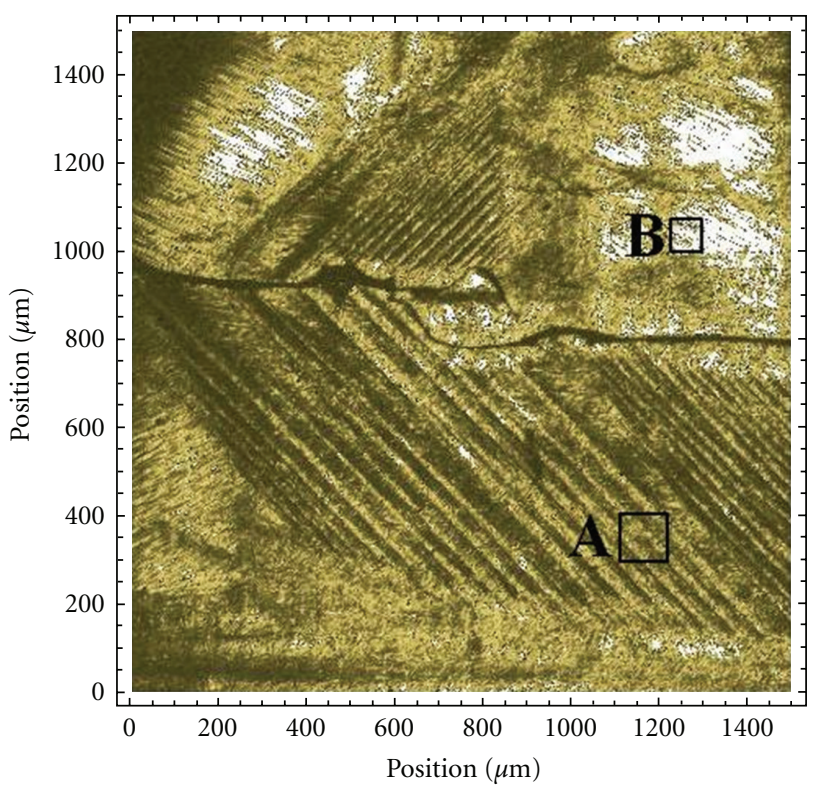

(a)

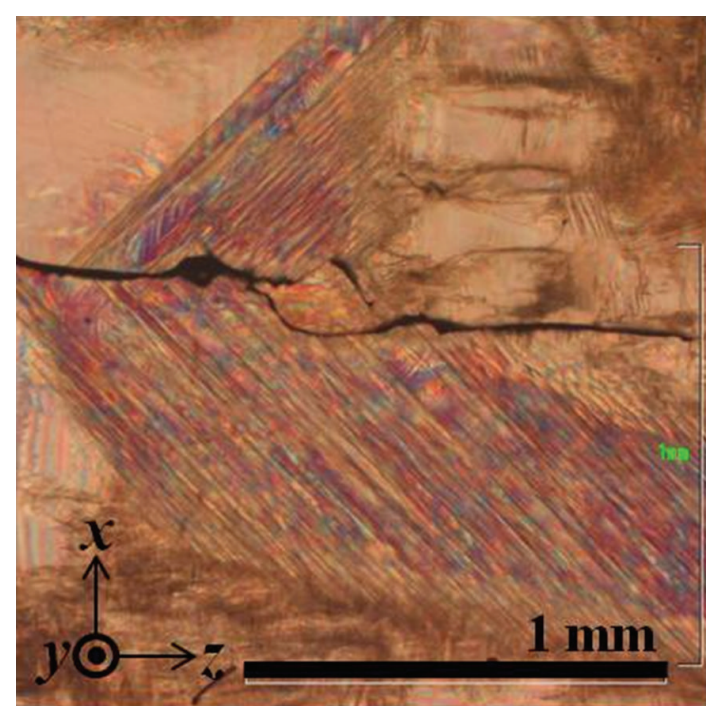

(b)

Figure 6: SHG image of the PZN-9PT (010) c plate (a) and corresponding polarization microscope image (b) [20].

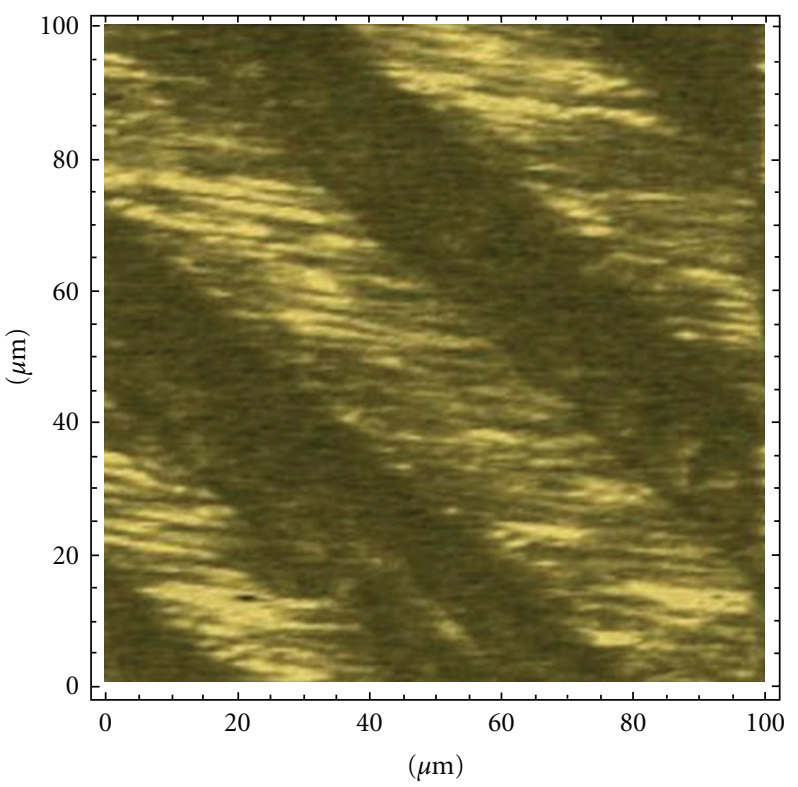

(a)
6\% \%0\%\&\&8000 8. $8500 \% 2800$ \&8 800,00\%8\% \&\&\&880008\%

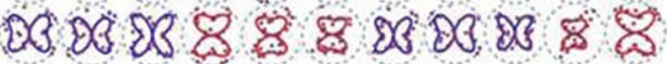
$00008 \% 8 \% 2038$ $\$ 03000300 \% 8$ \& 80303 $\$ 8 \% 00008 \% 8 \%$ क $8802008 \% 8 \%$ $000088 \& 00008 \& 8$ $8000 \% 8 \approx 000008$

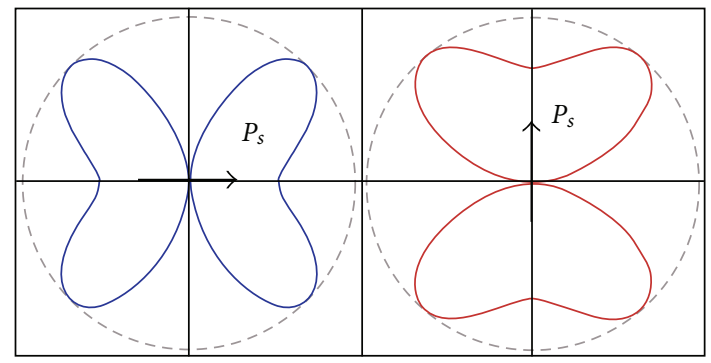

(b)

(c)

FIgURE 7: (a) Indicates the fine scanned SHG image of the region A in Figure 6(a). (b) shows the PolD map corresponding to the SHG image (a). Small dots indicate the experimental data and red and blue solid lines are the fitting curves. (c) shows calculated PolDs with (11)-(13) assuming the monoclinic symmetry $\mathrm{Pm}[20]$. 


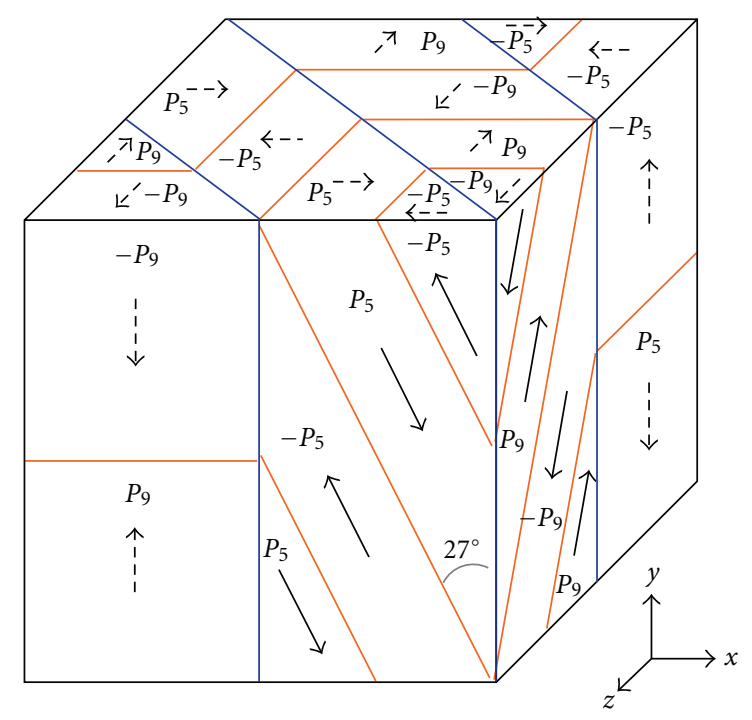

Figure 8: Domain configuration of a PZN/9PT single crystal deduced from the PolD maps [20].

red lines, and these two patterns almost exactly coincide with the SHG image of Figure 7(a). PolDs calculated with (11)(13) assuming monoclinic Pm are shown in Figure 7(c). Experimental results are in good agreement with the theory. Furthermore, the direction of $P_{s}$ can be estimated by the polar diagram and are shown in Figure $7(\mathrm{c})$. It is also revealed that the direction of $P_{s}$ is not located in this plane and the arrows represent the projection of $P_{s}$ on the (100) plane.

The same procedure is applied to region $\mathrm{B}$. In this case, only one pattern was observed in the polar diagram, and the form can be also explained with monoclinic symmetry. Full analyses of the PolD maps reveal the complicate domain structures as shown in Figure 8. Thus we conclude that the macroscopic symmetry of the MPB of PZN/9PT is monoclinic Pm. We would stress that the mapping of the polar diagrams can provide useful and quantitative information on the complex domain configuration.

\subsection{History Dependence and Time Evolution of the Order Pa-} rameter in $K L T$. $\mathrm{KTaO}_{3}$ is known to be quantum paraelectrics where a ferroelectric phase transition does not take place although a dielectric constant increases with decreasing temperature and attains high value at low temperature. With a partial substitution of Li ions at $\mathrm{K}$ sites, a dielectric peak appears at low temperature and the peak temperature shifts to higher temperature with increasing $\mathrm{Li}$ concentration. However, the polar state at low temperature had not been well recognized $[27,28]$. Yong et al. carried out dielectric and neutron diffuse scattering experiments and claimed that KLT is a relaxor because of the dielectric dispersions and diffuse scatterings related to the existence of polar nanoregions (PNRs) [29]. However, more convincing experimental results are needed on this point. To clarify the relaxor nature, we performed precise SHGM observations of KLT [2, 3].

We carried out the experiments using ICCD SHGM which enables to observe the $2 \mathrm{D}$ polarization distributions in

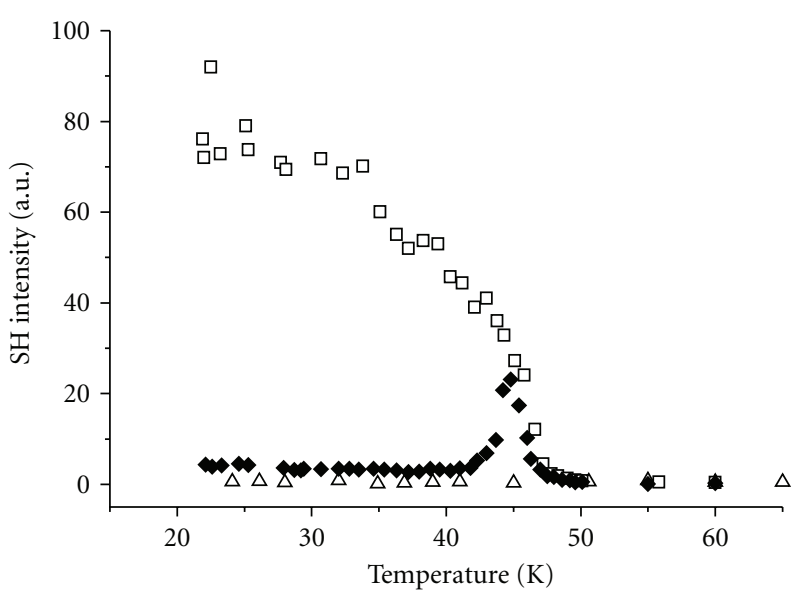

$\triangle \mathrm{ZFH} / \mathrm{ZFC}$
$\square \mathrm{FH} / \mathrm{FC}$
$\bullet \mathrm{FH} / \mathrm{ZFC}$

(a)

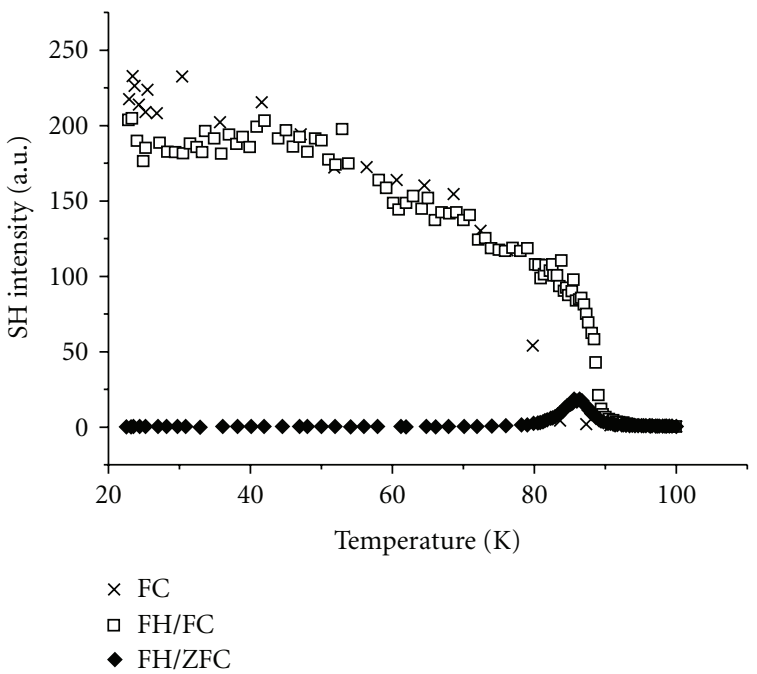

(b)

FIGURE 9: Temperature dependences of SHG intensities of KLT$2.6 \%$ (a) and KLT-6.8\% (b) [2].

a sample from the $\mathrm{SH}$ wave intensity distribution. The measurements were performed in the following five processes; (i) zero field cooling (ZFC), (ii) zero field heating after ZFC (ZFH/ZFC), (iii) field heating after ZFC (FH/ZFC), (iv) field cooling (FC), and (v) FH after FC (FH/FC). The electric field of $100 \mathrm{~V} / \mathrm{mm}$ was applied along the [001] direction and obtained results are shown in Figure 9. In ZFC and $\mathrm{ZFH} / \mathrm{ZFC}$ processes, no marked $\mathrm{SH}$ intensity is observed in the measurement temperature region. In FC, $\mathrm{SH}$ intensity starts to increase drastically at a certain temperature. The temperature at which $\mathrm{SH}$ intensity begins to increase shifts to higher temperature with increasing Li concentration. In $\mathrm{FH} / \mathrm{FC}$ process, $\mathrm{SH}$ intensity generated from the specimen at low temperature decreases with raising temperature and vanishes around a temperature $T_{p}$. In FH/ZFC, SH intensity dose not appear at low temperature even applying an 
electric field. With heating the sample, a weak SH intensity observed at low temperature starts to increase abruptly at certain temperature $T_{1}$ (42 K for KLT-2.6\% and $83 \mathrm{~K}$ for KLT-6.8\%), shows a peak and decreases, and finally vanishes at a temperature $T_{2} . T_{2}$ coincides with $T_{p}$ where $\mathrm{SH}$ intensity disappears in $\mathrm{FH} / \mathrm{FC}$ process. Based upon these results, we confirm the marked history dependence of the order parameter in KLT, which is a typical feature of relaxors.

In ferroelectrics, the domain switching time is known to be several $\mu \mathrm{sec}$. However, in relaxor, a relaxation time to the equilibrium state under external stimuli is several ten minutes. To observe this slow kinetics of the order parameter in KLT, SHGM observations were carried out in the following thermal procedure [30]. First the specimen was cooled down to $24 \mathrm{~K}$ without an electric field and heated up without an electric field to a certain temperature. When a temperature becomes stable, the electric field was applied and the $\mathrm{SH}$ intensity was measured as a function of time. Figure 10 shows the time dependence of the SH intensity at several temperatures observed in KLT-2.8\%. The present results indicate that the slow kinetics observed in $\mathrm{SH}$ intensity strongly depends on the temperature where an electric field is applied. The time evolution of SH intensity shows a drastic difference around $40 \mathrm{~K}$. Below $40 \mathrm{~K}$, SH intensity develops after a long incubation time. On the contrary, $\mathrm{SH}$ intensity shows a fast evolution followed by the slow process above $40 \mathrm{~K}$. This phenomenon could be explained by introducing a long-range strain field proposed by Chandra, who explained a long domain switching time observed in $\mathrm{BaTiO}_{3}$ [31]. In the case of KLT, off-centered Li ion could produce the longrange strain field. Thus above-mentioned two experimental results concerning the behavior of the order parameter, that is, the history dependence and the time evolution with a long relaxation time, confirm that KLT is relaxor.

4.4. Muscle Fibers. Muscles are functional body tissues and have structural hierarchy as shown in Figure 11: a muscle consists of bundles of muscle fibers of several ten $\mu \mathrm{m}$ diameter and a muscle fiber is an assemblage of myofibrils of several $\mu \mathrm{m}$ diameter. A myofibril is composed of a one-dimensional sequence of sarcomere with the period of about $2 \mu \mathrm{m}$ along the fiber direction. A sarcomere is a unit structure of muscle and contains thick myosin filaments, actin filaments, and titins that connect myosin filaments with sarcomere wall $(z$ line). Contraction and elongation of muscles are caused by relative sliding of actin and myosin fibers. X-ray and electron microscope revealed that a myosin filament is formed by coiled coil dimmers characterized by two myosin heads. The myosin chain is a coiled portion of two alpha helices, and $\mathrm{SH}$ waves are generated by the asymmetric charge distribution in hydrogen bonds in alpha helices. It is interesting to note that this spiral structure is opposite with respect to M-band which separates the sarcomere into two parts. Thus it is expected to have antiparallel polarization structure, or we can say, anti-ferroelectric structure in mesoscopic scale. However, no observation has been performed on this possible polar structure as yet. This is our observation motivation of myofibril using our SHG interference microscope.

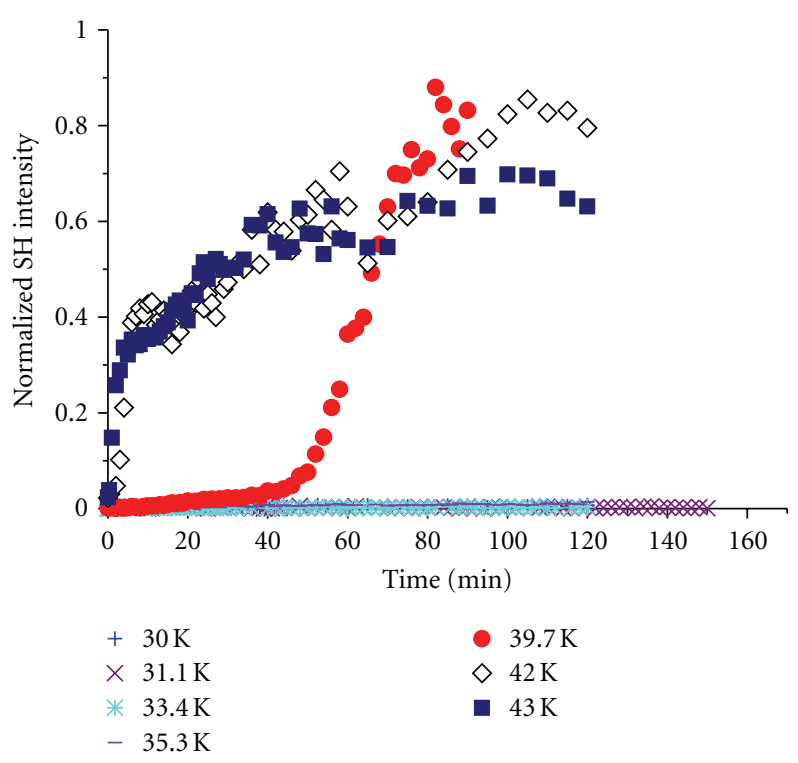

Figure 10: Time evolutions of SHG intensities of KLT under an electric field of $80 \mathrm{~V} / \mathrm{mm}$. The SH intensity at each temperature is normalized to the saturated value [30].

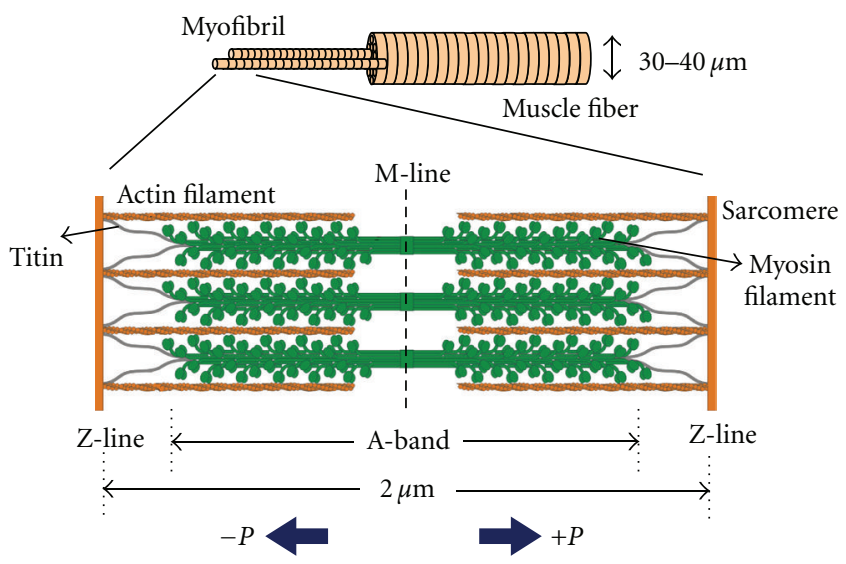

Figure 11: Structural hierarchy of a muscle.

For SHGM observation, we used white skeletal muscles, or psoas muscle of rabbit. Figure 12(a) shows an SHG image of muscle fibers. It is shown that muscle fibers are formed by regular assembly of the bright part. Figure 12(b) shows an SHG image of a myofibril extracted from muscle fibers by centrifuge. The periodicity of the bright parts is exactly same as that of muscle fiber, and finer scanning reveals $2 \mu \mathrm{m}$ periodicity of the bright parts. This fact indicates that the bright part corresponds to the A-band in sarcore. It is also interesting that muscle fibers shown in Figure 12(a) are a coherent bundle of myofibrils.

To reveal the polarity of sarcomere, we exploit the SH interference. The result is shown in Figure 13. Here the relative phase of $\mathrm{SH}$ waves from the sample and the reference plate is changed by a phase modulater. The most left image shows SHG image without interference, the next one from 


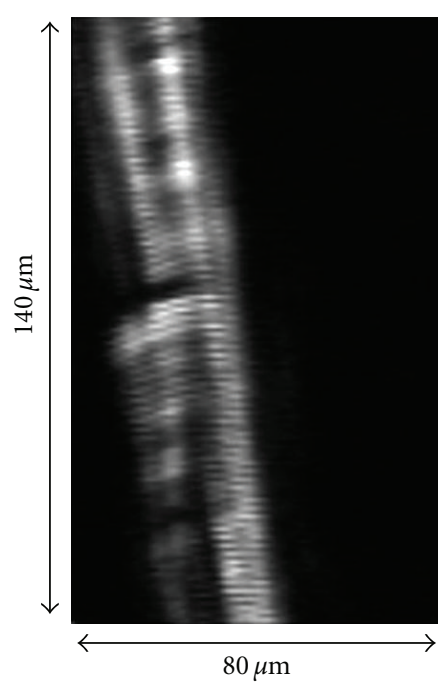

SHGIM image of muscle fiber

- Step $1 \mu \mathrm{m}$

- $140 \times 80 \mu \mathrm{m}$

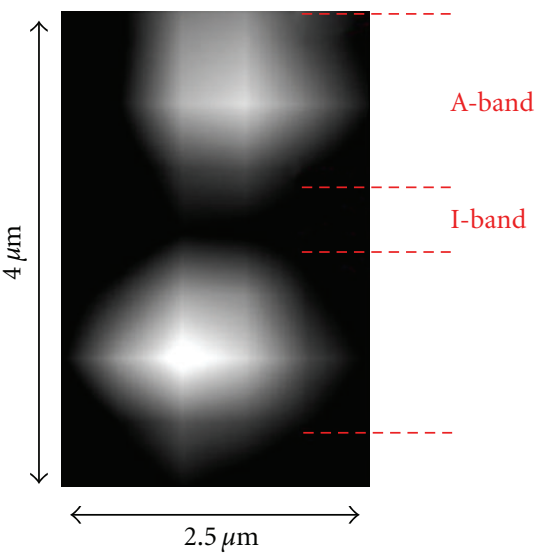

SHGIM image of muscle myofibril

- Step $0.5 \mu \mathrm{m}$

- $4 \times 2.5 \mu \mathrm{m}$

(a)

(b)

FIGURE 12: SHG images of muscle fiber (a) and myofibril (b).

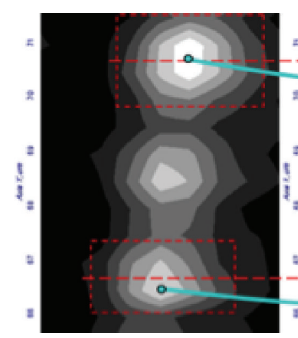

(a)

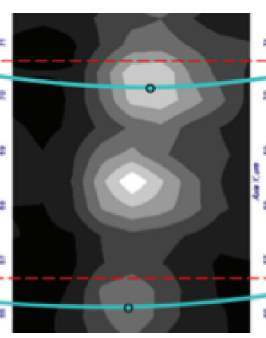

(b)

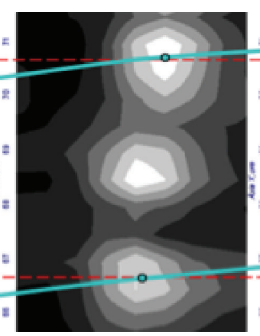

(c)

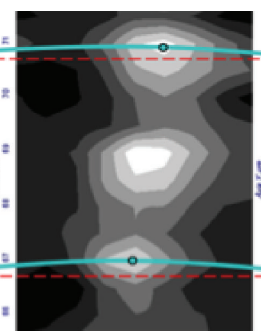

(d)

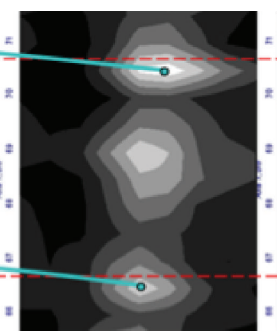

(e)

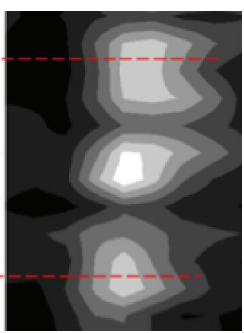

(f)

FIGURE 13: SHG interference images of myofibril. (a) shows SH image without the interference with reference SH waves. Images (b) to (f) are obtained by the interference by varying the phase difference between $0, \pi / 2, \pi, 3 \pi / 2$, and $\pi$, respectively.

the left is an image with phase difference 0 , then phase shift $\pi / 2, \pi, 3 \pi / 2$, and $2 \pi$. The position of the brightest part periodically shifts depending on the phase with periodicity of $2 \pi$. This means that the sarcomere consists of two parts with opposite polarities, and as a total, the myofibril has a periodically inverted polarization structure. It should be pointed out that the dimension of inverted part is about $1 \mu \mathrm{m}$, which is almost the limit of spatial resolution. Therefore the two parts with opposite polarization cannot be observed separately.

It is interesting that nature produces PPD structure in muscle fibers. If we consider this fact from an engineering perspective, it is really the QPM device, when the laser beam is introduced along the fiber axis. The periodicity of $2 \mu \mathrm{m}$ could provide the QPM device at the violet wavelength region. Actually the diameter of muscle fiber of $100 \mu \mathrm{m}$ is almost similar to an optical fiber and that of myofibril of several $\mu \mathrm{m}$ to a core of an optical fiber.

\section{Summary: Future Trend of the SHG Microscopy}

Due to the high sensitivity to the breaks of spatial inversion and time reversal symmetries, it is more and more recognized that the SHGM becomes a powerful method to investigate the nature of ferroic materials, that is, the behavior of order parameters, domain structures, symmetry changes, and so forth. It should be also pointed out that SHGM has been adopted not only for the material science but also for the biological and medical field $[32,33]$.

One of the bottle necks of SHGM is its spatial resolution. Using the conventional optical geometry, the spatial resolution is limited by the diffraction limit that is about the wavelength (several hundred $\mathrm{nm}$ ) of the incident waves. One way to overcome this limitation is the use of a nearfield microscope (SNOM). The SNOM has a long research history and the basic idea was already proposed by Synge in 
1928 [34]. Ash and Nicholls demonstrated experimentally the super-resolution at microwave frequencies [35]. Since then, the SNOM has been extended to the visible wavelength region and the resolution reaches $25 \mathrm{~nm}$ [36].

The techniques of SNOM can be divided into two categories dependent on a probe. One is aperture mode whose probe is used either to deliver the incident wave or collect the scattered light. The spatial resolution of this system is determined by the size of the aperture. Another way is called as an apertureless mode. This technique utilizes the nearfield waves scattered by a probe and the scattered wave is detected by a photomultiplier.

In recent years, SNOM has been also applied to the SHG. The first experimental result was reported by Smolyaninov et al. concerning the domain structure on Ni single crystal [37]. They used the aperture near-field scanning technique with an uncoated tapered fiber tip as a probe and achieved the resolution around $150 \mathrm{~nm}$. The apertureless SNOM SHG system was also constructed based on the atomic force microscope and realized fast-time resolution measurement [38]. The measurement of local variations of $d$-constants components is also reported [39]. The SNOM-SHGM is promising technique with high spatial resolution to investigate nanosize domain structures in thin films and the structural and physical properties of domain boundaries. However, extremely low intensity of the signal causes the low signal to noise ratio. Further improvements are required to establish the SNOMSHGM as a universal probe microscope.

\section{Acknowledgments}

The authors are grateful to Professor S. Ishiwata, Waseda University for preparing living samples of muscle fibers and myofibrils and a lot of valuable comments on the SHGM observations of them. This work is partly supported by the Grants-in-aid for Scientific Research (C) of MEXT, Japan and the special grants for research (A) and (B) in Waseda University. J. Kaneshiro is grateful for the support of Grantsin-Aid for JSPS Fellows. H. Yokota is thankful to the support of Career-Support Program for Woman Scientists at Chiba University.

\section{References}

[1] S. Arahira and Y. Uesu, "Optical second-harmonic generation in ferroelectric dicalcium lead propionate," Journal of the Physical Society of Japan, vol. 60, no. 7, pp. 2461-2469, 1991.

[2] H. Yokota, T. Oyama, and Y. Uesu, "Second-harmonic-generation microscopic observations of polar state in Li-doped $\mathrm{KTaO}_{3}$ under an electric field," Physical Review B, vol. 72, no. 14, Article ID 144103, 6 pages, 2005.

[3] H. Yokota and Y. Uesu, "Second-harmonic generation and Xray diffraction studies of the pretransitional region and polar phase in relaxor $\mathrm{K}_{(1-x)} \mathrm{Li}_{x} \mathrm{TaO}_{3}$," Brain and Nerve, vol. 75, no. 18, Article ID 184113, 8 pages, 2007.

[4] U. Pustogowa, T. A. Luce, W. Hübner, and K. H. Bennemann, "Theory of nonlinear magneto-optics (invited)," Journal of Applied Physics, vol. 79, no. 8, pp. 6177-6180, 1996.

[5] N. Ogawa, T. Satoh, Y. Ogimoto, and K. Miyano, "Nonlinear optical detection of a ferromagnetic state at the single interface of an antiferromagnetic $\mathrm{LaMnO}_{3} / \mathrm{SrMnO}_{3}$ double layer," Physical Review B, vol. 78, no. 21, Article ID 212409, 4 pages, 2008.

[6] M. Fiebig, T. Lottermoser, D. Fröhlich, A. V. Goltsev, and R. V. Pisarev, "Observation of coupled magnetic and electric domains," Nature, vol. 419, no. 6909, pp. 818-820, 2002.

[7] M. Fiebig, V. V. Pavlov, and R. V. Pisarev, "Second-harmonic generation as a tool for studying electronic and magnetic structures of crystals: review," Journal of the Optical Society of America B, vol. 22, no. 1, pp. 96-118, 2005.

[8] M. Fiebig, "Magnetoelectric phase control in multiferroic manganites," Phase Transitions, vol. 79, no. 12, pp. 947-956, 2006.

[9] B. B. Van Aken, J. P. Rivera, H. Schmid, and M. Fiebig, "Observation of ferrotoroidic domains," Nature, vol. 449, no. 7163, pp. 702-705, 2007.

[10] Y. Uesu, K. Yamane, and B. A. Strukov, "Optical second-harmonic generation of ammonium fluoroberyllate crystal in the incommensurate and ferroelectric phases," Japanese Journal of Applied Physics, vol. 28, no. 3, pp. 453-456, 1989.

[11] W. Kleemann and J. Dec, "Comment on "Critical behavior of ferroelectric $\mathrm{SrTi}^{18} \mathrm{O}_{3}$ ”," Physical Review B, vol. 75, Article ID 027101, 3 pages, 2007.

[12] Y. Uesu, S. Kurimura, and Y. Yamamoto, "Optical second harmonic images of 90 domain structure in $\mathrm{BaTiO}_{3}$ and periodically inverted antiparallel domains in $\mathrm{LiTaO}_{3}$," Applied Physics Letters, vol. 66, pp. 2165-2167, 1995.

[13] S. Kurimura and Y. Uesu, "Application of the second harmonic generation microscope to nondestructive observation of periodically poled ferroelectric domains in quasi-phase-matched wavelength converters," Journal of Applied Physics, vol. 81, no. 1, pp. 369-375, 1997.

[14] N. Kato, K. Saito, H. Aida, and Y. Uesu, "Observations of merocyanine J-aggregate domains in mixed molecular monolayers using SHG/fluorescence and atomic force microscopes," Chemical Physics Letters, vol. 312, no. 2-4, pp. 115-120, 1999.

[15] S. A. Denev, T. A. Lummen, E. Barnes, A. Kumar, and V. Gopalan, "Probing Ferroelectrics Using Optical Second Harmonic Generation," Journal of the American Ceramic Society, vol. 94, pp. 2699-2727, 2011.

[16] G. D. Boyd and D. A. Kleinman, "Parametric interaction of focused Gaussian light beams," Journal of Applied Physics, vol. 39, no. 8, pp. 3597-3639, 1968.

[17] Y. Uesu, H. Yokota, S. Kawado, J. Kaneshiro, S. Kurimura, and N. Kato, "Three-dimensional observations of periodically poled domains in a $\mathrm{LiTaO}_{3}$ quasiphase matching crystal by second harmonic generation tomography," Applied Physics Letters, vol. 91, no. 18, Article ID 182904, 3 pages, 2007.

[18] J. Kaneshiro, Y. Uesu, and T. Fukui, "Visibility of inverted domain structures using the second harmonic generation microscope: comparison of interference and non-interference cases," Journal of the Optical Society of America B, vol. 27, no. 5, pp. 888-894, 2010.

[19] J. Kaneshiro, S. Kawado, H. Yokota, Y. Uesu, and T. Fukui, "Three-dimensional observations of polar domain structures using a confocal second-harmonic generation interference microscope," Journal of Applied Physics, vol. 104, no. 5, Article ID 054112, 7 pages, 2008.

[20] J. Kaneshiro and Y. Uesu, "Domain structure analysis of $\mathrm{Pb}\left(\mathrm{Zn}_{1 / 3} \mathrm{Nb}_{2 / 3}\right) \mathrm{O}_{3}-9 \% \mathrm{PbTiO}_{3}$ single crystals using optical second harmonic generation microscopy," Physical Review B, vol. 82, no. 82, Article ID 184116, 8 pages, 2010. 
[21] V. Grubsky, S. MacCormack, and J. Feinberg, "All-optical three-dimensional mapping of 180 domains hidden in a $\mathrm{BaTiO}_{3}$ crystal," Optics Letters, vol. 21, no. 1, pp. 6-8, 1996.

[22] Y. Uesu, H. Shibata, S. Suzuki, and S. Shimada, "3D images of inverted domain structure in $\mathrm{LiNbO}_{3}$ using SHG interference microscope," Ferroelectrics, vol. 304, no. 1, pp. 99-103, 2004.

[23] B. Jaffe, W. R. Cook, and H. Jaffe, Piezoelectric Ceramics, Academic Press, London, UK, 1971.

[24] K. Fujishiro, R. Vlokh, Y. Uesu et al., "Optical observation of heterophase and domain structures in relaxor ferroelectrics $\mathrm{Pb}\left(\mathrm{Zn}_{1 / 3} \mathrm{Nb}_{2 / 3}\right) \mathrm{O}_{3} / 9 \% \mathrm{PbTiO}_{3}$," Japanese Journal of Applied Physics, vol. 37, no. 9, pp. 5246-5248, 1998.

[25] B. Noheda, D. E. Cox, G. Shirane, J. A. Gonzalo, L.-E. Cross, and S. E. Park, "A monoclinic ferroelectric phase in the $\mathrm{Pb}\left(\mathrm{Zr}_{1-x} \mathrm{Ti}_{x}\right) \mathrm{O}_{3}$ solid solution," Applied Physics Letters, vol. 74, no. 14, pp. 2059-2061, 1999.

[26] H. Fu and R. E. Cohen, "Polarization rotation mechanism for ultrahigh electromechanical response in single-crystal piezoelectrics," Nature, vol. 403, no. 6767, pp. 281-283, 2000.

[27] U. T. Hochli, H. E. Weibel, and L. A. Boatner, "Stabilisation of polarised clusters in $\mathrm{KTaO}_{3}$ by Li defects: formation of a polar glass," Journal of Physics C, vol. 12, no. 14, article L563, 1979.

[28] R. L. Prater, L. L. Chase, and L. A. Boatner, "Raman scattering studies of the impurity-induced ferroelectric phase transition in $\mathrm{KTaO}_{3}$ : Li," Physical Review B, vol. 23, no. 11, pp. 5904$5915,1981$.

[29] G. Yong, J. Toulouse, R. Erwin, S. M. Shapiro, and B. Hennion, "Pretransitional diffuse neutron scattering in the mixed perovskite relaxor $\mathrm{K}_{1-x} \mathrm{LixTaO}_{3}$," Physical Review B, vol. 62, no. 22, pp. 14736-14743, 2000.

[30] H. Yokota and Y. Uesu, "Extremely slow time evolution of the order parameter under an electric field in relaxor $\mathrm{K}_{0.97} \mathrm{Li}_{0.03} \mathrm{TaO}_{3}$," Journal of Physics, vol. 19, no. 10, Article ID 102201, 2007.

[31] P. Chandra, "Nucleation in the presence of long-range interactions," Physical Review A, vol. 39, no. 7, pp. 3672-3681, 1989.

[32] S. V. Plotnikov, A. C. Millard, P. J. Campagnola, and W. A. Mohler, "Characterization of the myosin-based source for second-harmonic generation from muscle sarcomeres," Biophysical Journal, vol. 90, no. 2, pp. 693-703, 2006.

[33] M. E. Llewellyn, R. P. J. Barretto, S. L. Delp, and M. J. Schnitzer, "Minimally invasive high-speed imaging of sarcomere contractile dynamics in mice and humans," Nature, vol. 454, no. 7205, pp. 784-788, 2008.

[34] E. H. Synge, "A suggested method for extending microscopic resolution into the ultra-microscopic region," Philosophical Magazine Series, vol. 6, no. 35, pp. 356-362, 1928.

[35] E. A. Ash and G. Nicholls, "Super-resolution aperture scanning microscope," Nature, vol. 237, no. 5357, pp. 510-512, 1972.

[36] D. W. Pohl, W. Denk, and M. Lanz, "Optical stethoscopy: image recording with resolution $\lambda / 20$," Applied Physics Letters, vol. 44, no. 7, p. 651, 1984.

[37] I. I. Smolyaninov, A. V. Zayats, and C. C. Davis, "Near-field second-harmonic imaging of ferromagnetic and ferroelectric materials," Optics Letters, vol. 22, no. 21, pp. 1592-1594, 1997.

[38] I. I. Smolyaninov, C. H. Lee, and C. C. Davis, "Near-field second harmonic imaging of lead zirconate titanate piezoceramic," Applied Physics Letters, vol. 74, no. 14, pp. 1942$1944,1999$.

[39] W. Dickson, S. Takahashi, C. M. I. Boronat, R. M. Bowman, J. M. Gregg, and A. V. Zayats, "Near-field second-harmonic imaging of thin ferroelectric films," Physical Review B, vol. 72, no. 9, Article ID 094110, 6 pages, 2005. 

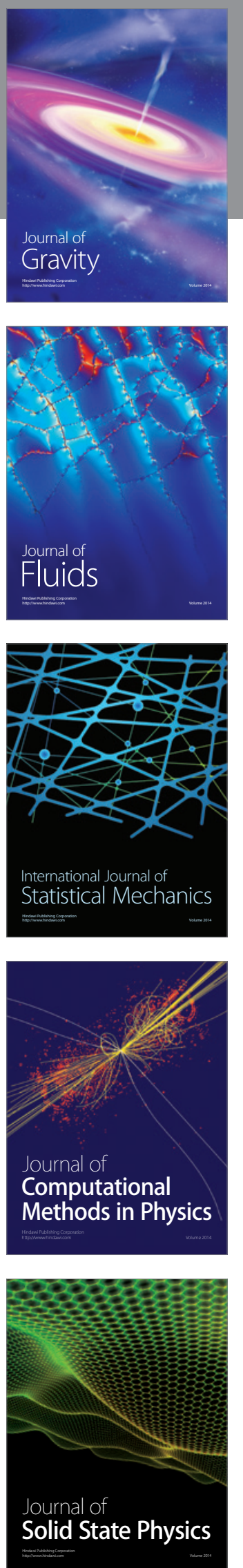

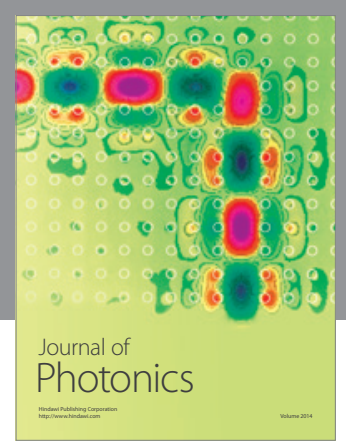

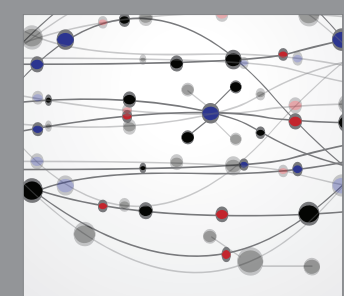

The Scientific World Journal
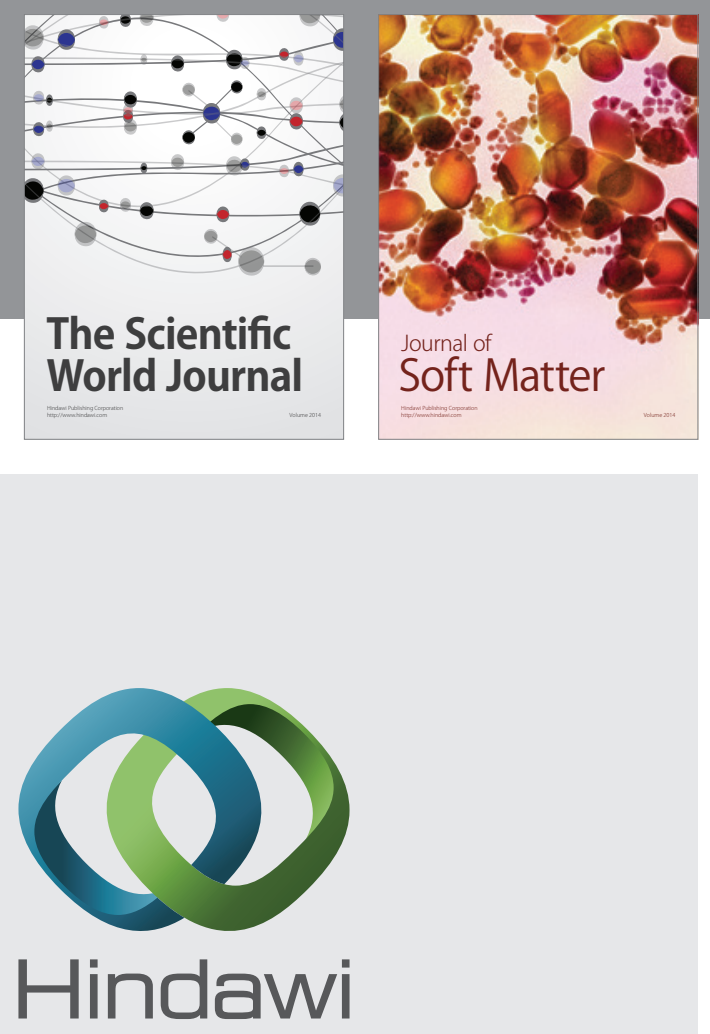

Submit your manuscripts at

http://www.hindawi.com
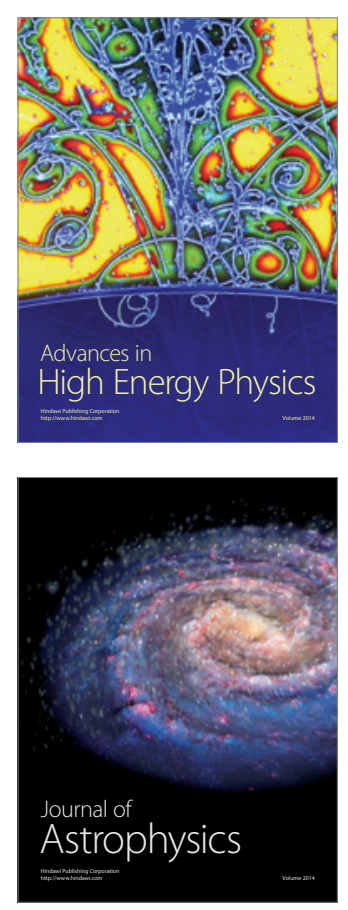
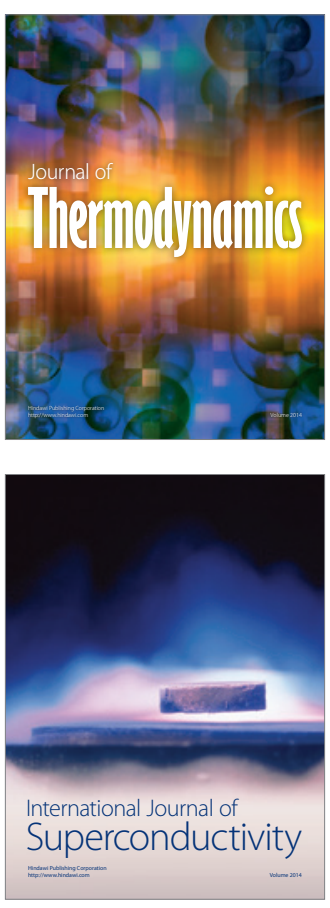
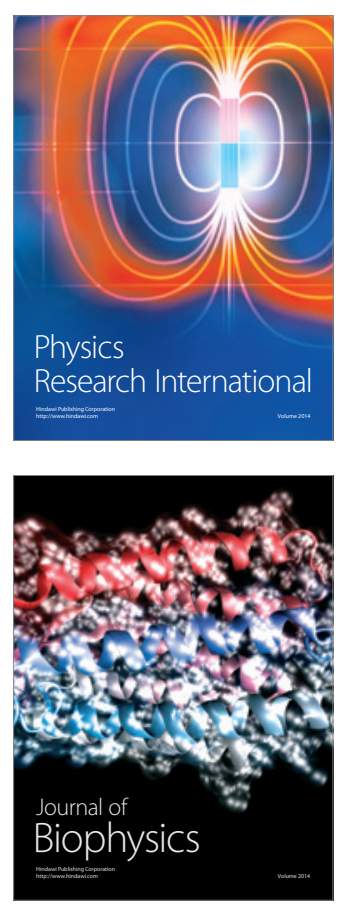
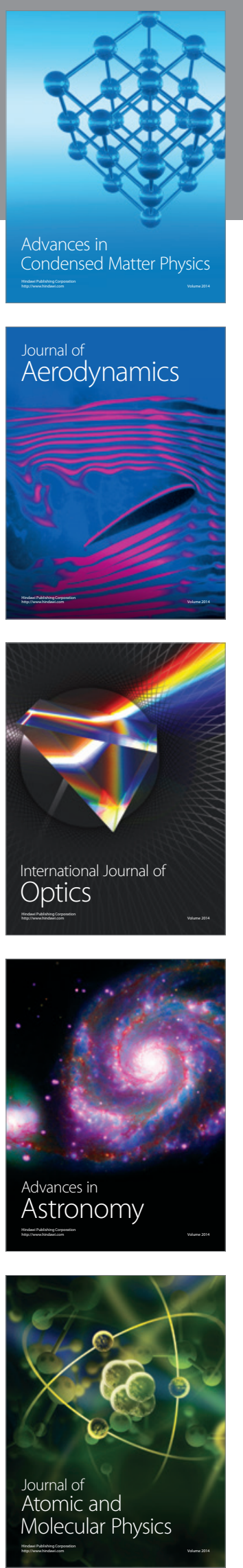